\title{
Community detection analysis in wind speed-monitoring systems using mutual information-based complex network
}

Cite as: Chaos 29, 043107 (2019); https://doi.org/10.1063/1.5054724

Submitted: 03 September 2018 . Accepted: 14 March 2019 . Published Online: 10 April 2019

Mohamed Laib (D), Fabian Guignard (D), Mikhail Kanevski (D), and Luciano Telesca
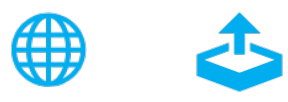

\section{ARTICLES YOU MAY BE INTERESTED IN}

Explosive synchronization in frequency displaced multiplex networks

Chaos: An Interdisciplinary Journal of Nonlinear Science 29, 041102 (2019); https://

doi.org/10.1063/1.5092226

Spike-burst chimera states in an adaptive exponential integrate-and-fire neuronal network Chaos: An Interdisciplinary Journal of Nonlinear Science 29, 043106 (2019); https:// doi.org/10.1063/1.5087129

Selection of spatiotemporal patterns in arrays of spatially distributed oscillators indirectly coupled via a diffusive environment

Chaos: An Interdisciplinary Journal of Nonlinear Science 29, 043104 (2019); https:// doi.org/10.1063/1.5058741 


\title{
Community detection analysis in wind speed-monitoring systems using mutual information-based complex network
}

\author{
Cite as: Chaos 29, 043107 (2019); doi: 10.1063/1.5054724 \\ Submitted: 3 September 2018 . Accepted: 14 March 2019. \\ Published Online: 10 April 2019
}

Mohamed Laib, ${ }^{1, \text { a) }}$ (D) Fabian Guignard, ${ }^{1}$ (D) Mikhail Kanevski, ${ }^{\text {(I) }}$ and Luciano Telesca ${ }^{2}$

\begin{abstract}
AFFILIATIONS
${ }^{1}$ IDYST, Faculty of Geosciences and Environment, University of Lausanne, 1015 Lausanne, Switzerland

${ }^{2} \mathrm{CNR}$, Istituto di Metodologie per l'Analisi Ambientale, 85050 Tito, PZ, Italy
\end{abstract}

a) Electronic mail: Mohamed.Laib@unil.ch

\begin{abstract}
A mutual information-based weighted network representation of a wide wind speed-monitoring system in Switzerland was analyzed in order to detect communities. Two communities have been revealed, corresponding to two clusters of sensors situated, respectively, on the Alps and on the Jura-Plateau that define the two major climatic zones of Switzerland. The silhouette measure is used to evaluate the obtained communities and confirm the membership of each sensor to its cluster.
\end{abstract}

Published under license by AIP Publishing. https://doi.org/10.1063/1.5054724

Since the installation of dense meteorological monitoring systems made available a huge amount of data, investigating the properties of meteoclimatic parameters has become challenging to understand the mechanisms underlying climatic systems. Complex networks represent an important theoretical framework that helps to describe and understand the interaction among meteoclimatic parameters concomitantly measured by sensors of a very dense monitoring system. This work proposes a mutual information-based network to study the interaction between the wind speed series measured by a meteorological monitoring system in Switzerland, characterized by so diverse topographies. Applying a multilevel community detection method, two clusters of wind stations were identified, matching the two main climatic zones of Switzerland. The results of this study suggest new methodological approaches to investigate wind speed time series.

\section{INTRODUCTION}

Over the past few years, more and more data have been being collected at ever higher frequencies that developing efficient patterndetection methods and data-mining techniques has become very crucial to identify a few highly informative features. In this context, one of the most relevant examples is given by high-dimensional (multiple) time series that originate from constituent units of large systems characterized by inner interactions.

The cooperative behavior within a complex system involving relationships among its constituent units can be effectively described by networks, where the interactions among the constituents (or nodes of the network) are represented by links. The topology of the network, which coincides with the topology of such interconnections or links, is in itself complex. ${ }^{3,25}$ These networks show a certain organization at a mesoscopic level, which is intermediate between the microscopic level that involves the single constituent units and the macroscopic level that involves the entire system as a whole. This mesoscopic level reflects the modular organization of the system, characterized by the existence of interconnected groups where some units are heavily linked with each other while, at the same time, are less correlated with the rest of the network. These interconnected groups are generally featured as communities. ${ }^{14,16}$ Detecting such communities represents an important step in the dynamical characterization of a network, because it could reveal special relationships between the nodes that may not be easily detectable by direct empirical tests: ${ }^{24}$ this helps to a better understanding of the characteristics of dynamic processes that take place in a network.

The use of complex networks to understand the interactions characterizing a climatic system has been growing in the past years, ${ }^{9,10,17,38,39}$ and various approaches have been used in constructing the related networks. ${ }^{10,34,37,38,44}$ Furthermore, a complex 


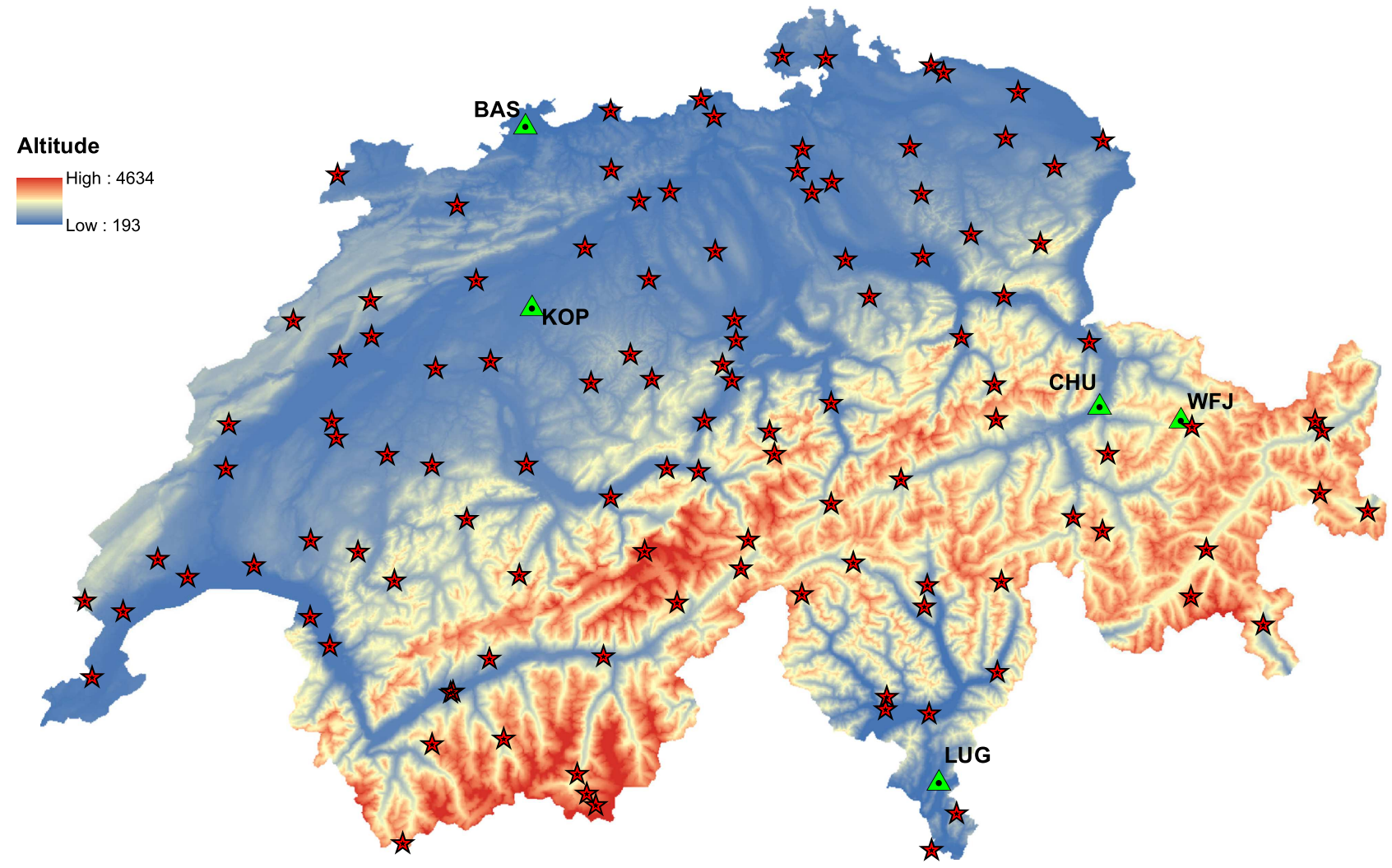

FIG. 1. Study area and location of measuring stations. The named stations are used as examples in Fig. 2 (see Table I for more details).

network offers a new mathmatical modeling approach for nonlinear dynamics ${ }^{11}$ and for climatological data analysis. ${ }^{1 .}$

Among the meteoclimatic parameters, wind is an important factor that influences the evolution of a climatic system; several studies have been devoted to understand better its time dynamics by using several methods, such as extreme value theory and copula, machine learning algorithms, ${ }^{36}$ visibility graph analysis, ${ }^{28}$ Markov chains models, ${ }^{18}$ fractal, ${ }^{13,27}$ and multifractal analysis.

The topological properties of wind systems have been a focus of investigation only in the very recent years. Laib et al. ${ }^{23}$ studied the long-range fluctuations in the connectivity density time series of a correlation-based network of high-dimensional wind speed time series recorded by a monitoring system in Switzerland. They found that the daily time series of a connectivity density of the wind speed network is characterized by a clear annual periodicity that modulates the connectivity density more intensively for low than high absolute values of the correlation threshold. Laib et al. ${ }^{22}$ analyzed the multifractality of connectivity density time series of the wind network and found that the larger multifractality at higher absolute values of thresholds could be probably induced by the higher spatial sparseness of the linked nodes at these thresholds.

Considering the topographic conditions of Switzerland and its widespread wind monitoring systems, it is challenging to investigate the topology of the wind network in terms of existence of network communities, and to check if these communities match with the topography of the territory.

To this aim, the edges of the network (the links between any two stations of the wind system, which are the nodes of the network) are weighted by the mutual information between the wind time series recorded at each station. The mutual information, which quantifies the degree of nonlinear correlation between two time series, has been already used to construct seismic networks, ${ }^{19}$ global foreign exchange markets, ${ }^{4}$ and prediction of stock market movements. ${ }^{20}$

\section{DATA AND NETWORK CONSTRUCTION}

The data used in this work consist of daily mean wind speed, collected from 119 measuring stations from 2012 to 2016 by SwissMetNet, which is one of the weather monitoring systems in Switzerland covering almost homogeneously all the Swiss territory (Fig. 1). Figure 2 shows, as an example, some of the measured wind speed series (Table I).

To construct the network, the mutual information was used as a metric to weight the edges between the nodes,

$$
I(X, Y)=\sum_{x \in X} \sum_{y \in Y} p(x, y) \log \left(\frac{p(x, y)}{p(x) p(y)}\right),
$$


WFJ

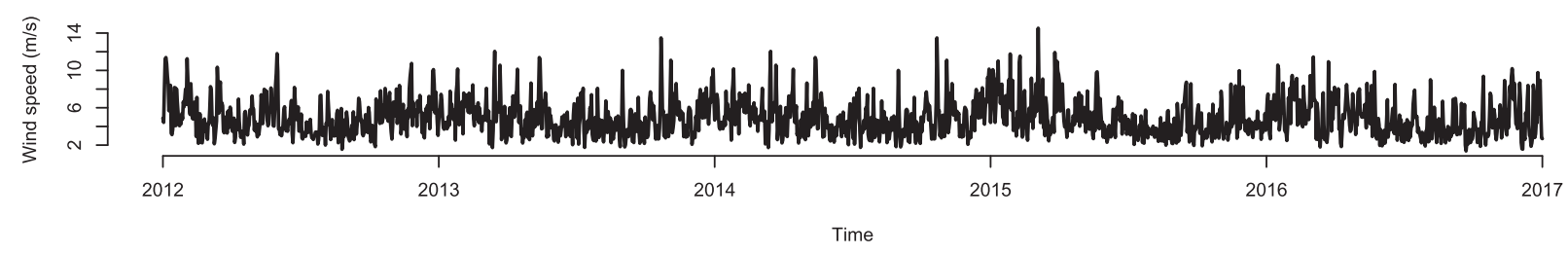

KOP

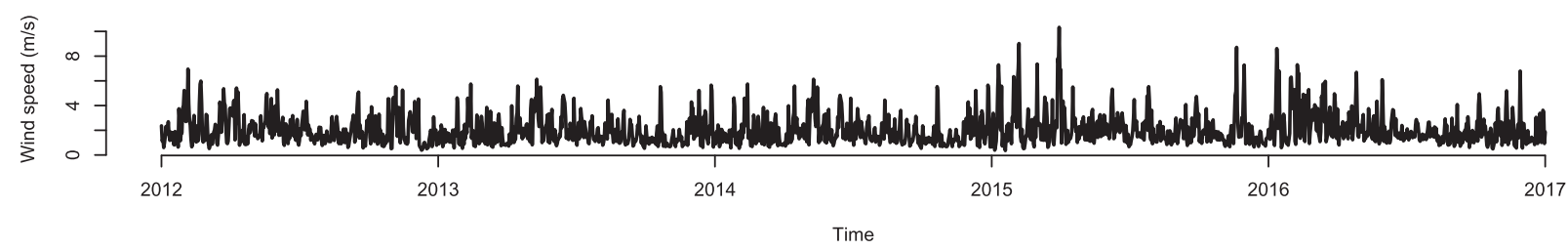

BAS

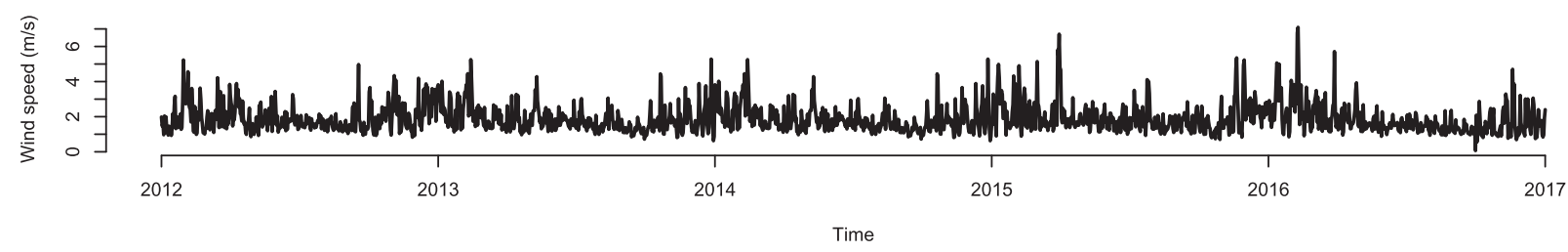

$\mathrm{CHU}$

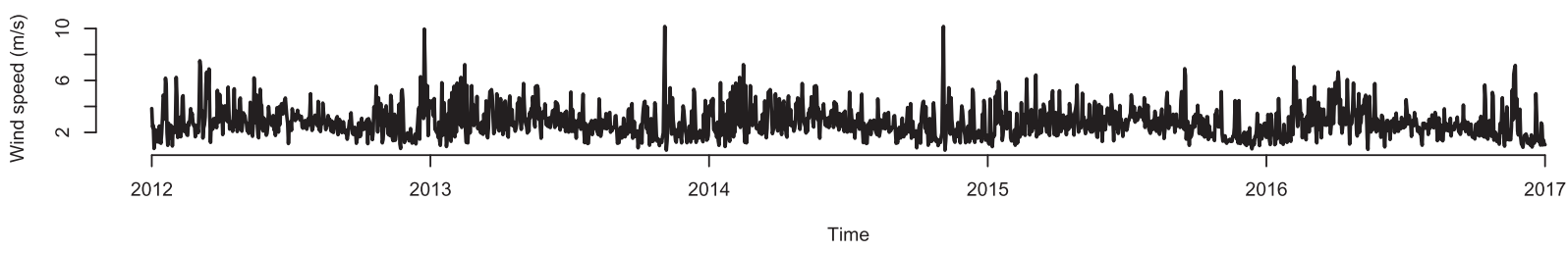

LUG

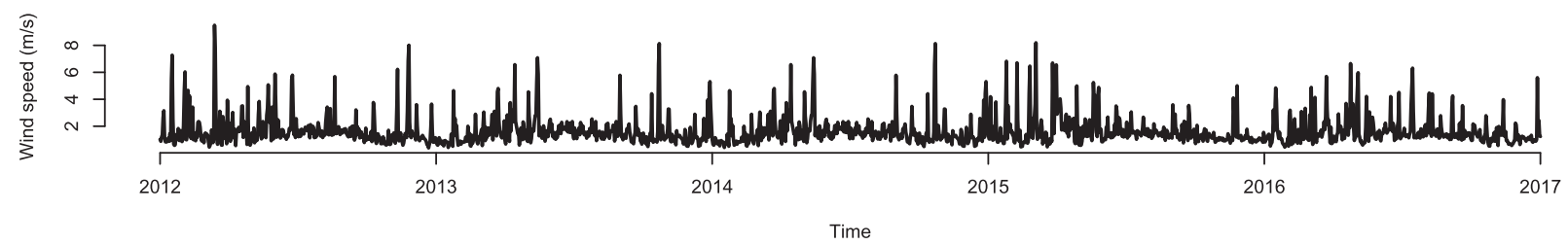

FIG. 2. Some example of daily wind time series.

where $X$ and $Y$ are two different random variables (wind time series), $p(x)$ and $p(y)$ are their respective probabilities, while $p(x, y)$ is their joint probability.

Mutual information is a measure of the amount of information that one random variable contains about another random variable.
It can be shown that Eq. (1) can be written as follows:?

$$
I(X, Y)=D(p(x, y) \| p(x) p(y)),
$$

where $D(\cdot \| \cdot)$ is the Kullback-Leibler divergence, which is a dissimilarity measure between two probability distributions. 
TABLE I. Brief information on the stations used as an example in Fig. 2.

\begin{tabular}{lccc}
\hline \hline Station & Name & Longitude/latitude & Altitude (m) \\
\hline WFJ & Weissfluhjoch & $9^{\circ} 48^{\prime} / 46^{\circ} 50^{\prime}$ & 2691 \\
KOP & Koppigen & $7^{\circ} 36^{\prime} / 47^{\circ} 07^{\prime}$ & 484 \\
BAS & Basel & $7^{\circ} 35^{\prime} / 47^{\circ} 32^{\prime}$ & 316 \\
CHU & Chur & $9^{\circ} 32^{\prime} / 46^{\circ} 52^{\prime}$ & 556 \\
LUG & Lugano & $8^{\circ} 58^{\prime} / 46^{\circ} 00^{\prime}$ & 273 \\
\hline \hline
\end{tabular}

Thus, the mutual information can be seen as the departure of the joint probability $p(x, y)$ from the product of the two marginal probabilities $p(x)$ and $p(y)$. We can easily show that $I(X, Y) \geqslant 0$ with equality if and only if $X$ and $Y$ are independent. ${ }^{7}$ Consequently, the higher the mutual information, the stronger the dependence between $X$ and $Y$.

In this work, the probabilities $p(x), p(y)$, and $p(x, y)$ are calculated by using kernel smoothing for density estimation. ${ }^{40,41}$

Since the mutual information, defined in Eq. (1), is symmetric, the network is undirected. Furthermore, the network is completely connected, because all the nodes are connected. However, the edges differ by their weights given by the mutual information.

\section{COMMUNITY DETECTION BY THE MULTILEVEL METHOD}

Proposed by Blondel et al., ${ }^{2}$ the MultiLevel algorithm (ML) is one of the community detection methods. Yang et al. ${ }^{45}$ compared several well-known algorithms of community detection (Edgebetweenness, ${ }^{16}$ Fastgreedy, ${ }^{5}$ Infomap, ${ }^{32}$ walktrap, ${ }^{29}$ and Spinglass ${ }^{31}$ ), and found that ML outperforms all other algorithms on a set of benchmarks.

The ML algorithm aims to optimise the modularity, ${ }^{26}$ which measures the density of links inside a community, and compares it between other communities. The modularity is defined as follows:

$$
Q=\frac{1}{2 m} \sum_{i j}\left[A_{i j}-\frac{k_{i} k_{j}}{2 m} \delta\left(c_{i}, c_{j}\right)\right],
$$

where $Q$ ranges between -1 and 1 and $^{2} A_{i j}$ is the weight between nodes $i$ and $j, 2 m$ is the sum of all the weights in the graph, $k_{i}$ and $k_{j}$ are the sum of weights connected to nodes $i$ and $j$, respectively, $c_{i}$ and $c_{j}$ communities (classes) of nodes. $\delta$ is the delta function of the variables $c_{i}$ and $c_{j}$.

The ML algorithm consists of two iterative steps. First, each node is considered as a community for an initial partition. Then, the node $i$ is removed from its community $c_{i}$ and placed in another community $c_{j}$, if this replacement maximises the modularity [Eq. (3)]; otherwise, the node $i$ remains in its original community until when there is no gain in the modularity. The gain in modularity of moving a node $i$ into a community $C$ is computed as follows:

$$
\begin{aligned}
\Delta Q= & {\left[\frac{\sum_{i n}+2 k_{i, i n}}{2 m}-\left(\frac{\sum_{t o t}+k_{i}}{2 m}\right)^{2}\right] } \\
& -\left[\frac{\sum_{i n}}{2 m}-\left(\frac{\sum_{t o t}}{2 m}\right)^{2}-\left(\frac{k_{i}}{2 m}\right)^{2}\right],
\end{aligned}
$$

where $\sum_{i n}$ is the sum of weights inside $C, \sum_{\text {tot }}$ is the sum of weights of edges incident to nodes in community $C, k_{i, i n}$ is the sum of weights of connection of node $i$ with other nodes of the same community $C$, and $m$ is the sum of all weights in the network.

The second step consists in building a new network whose nodes are now the communities found during the first step. The weights between these new nodes are defined by the sum of the link weights of the corresponding communities of the old network, as it is proposed by Arenas et al. ${ }^{1}$ for reducing the size of a complex network by preserving the modularity. Then, the first step is applied again on the new network iteratively until the modularity stops to increase.

\section{RESULTS AND DISCUSSION}

Figure 3 shows the mutual information among all the nodes. Applying the community detection based on the MultiLevel method, three different communities are identified, as shown in Fig. 4. Mapping the communities on the territory of Switzerland (Fig. 5), two classes are mixed spatially (stations indicated by green and black circles).

To quantify such spatial mixing effects, the well-known silhouette width was used. ${ }^{33}$ This is defined as

$$
s(i)=\frac{b(i)-a(i)}{\max \{a(i), b(i)\}},
$$

where $a(i)$ is the dissimilarity between the node (object) $i$ and the other nodes of the same community, $b(i)$ is the minimum value of dissimilarity between the node $i$ and the other nodes of other communities, and the dissimilarity is the minimum Euclidean distance. From Eq. (5), we can see that the silhouette $s(i)$ ranges between -1 and 1 .

Figure 6 shows the silhouette widths for each station of each community, by applying the silhouette on the mutual information matrix and the obtained communities. Figure 7 shows the silhouette

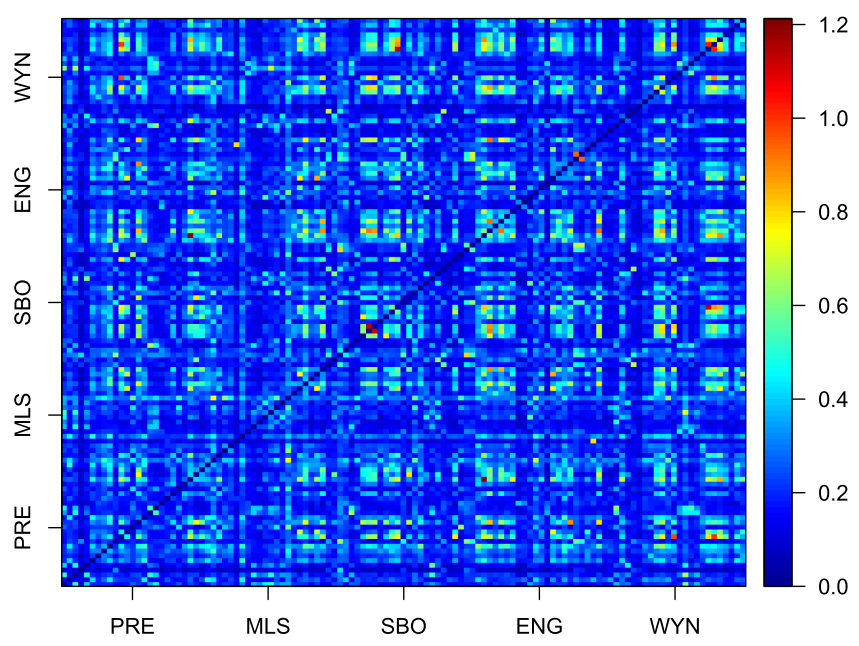

FIG. 3. Mutual information matrix $(119 \times 119)$. Each cell $(i, j)$ represents the mutual information between station $i$ and station $j$. 


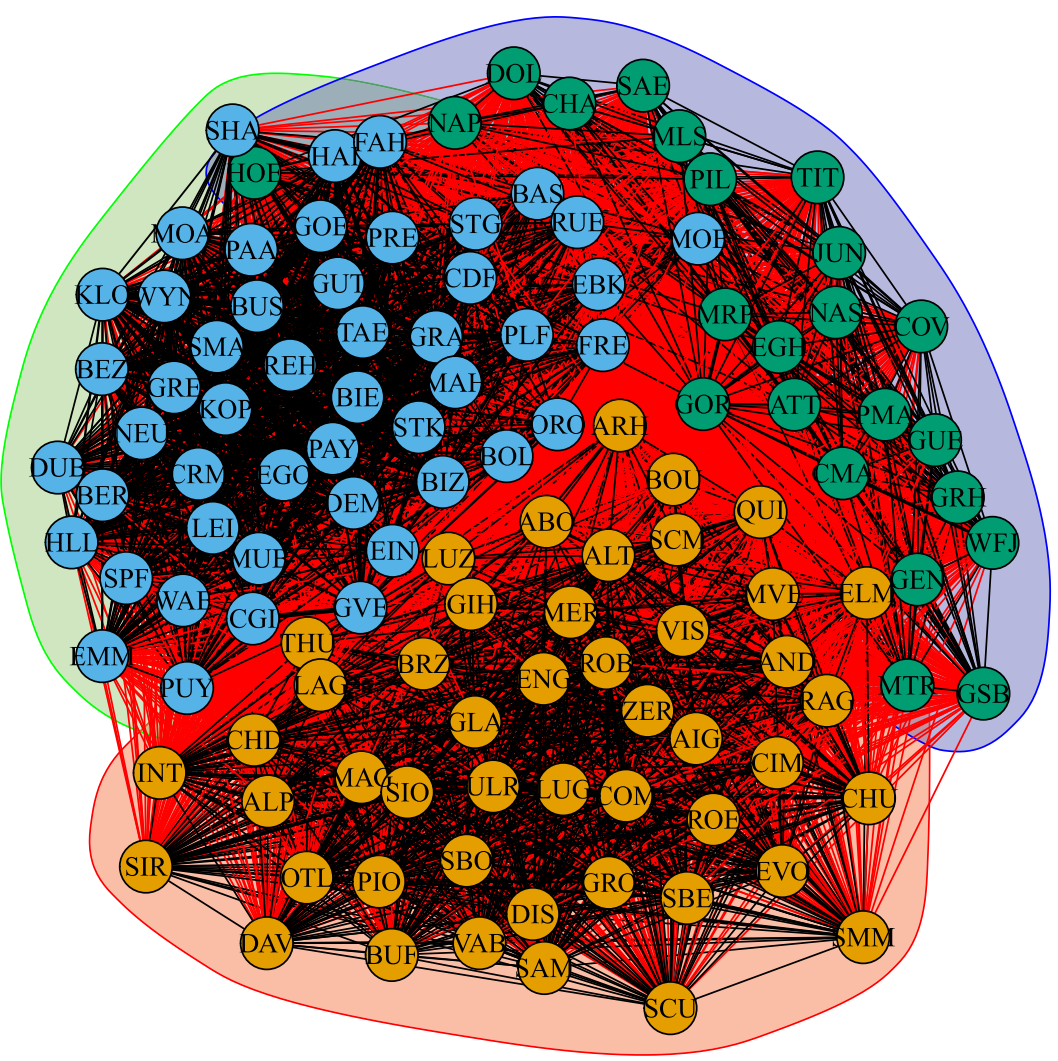

FIG. 4. Network visualisation with the three communities obtained by the ML method before applying the Seasonal Decomposition of Time Series by Loess (STL).

widths, by applying it on the XY coordinates. The average values are 0.19 (Mutual information matrix) and 0.09 (XY coordinates). These low values indicate that the obtained communities are not well spatially separated.
In order to understand the origin of such spatial mixing between communities, we filtered out from the wind series the trend and the yearly cycle ${ }^{21}$ by using the Seasonal Decomposition of Time Series by Loess (STL) ${ }^{6}$ (implemented by using the stl function of the stats

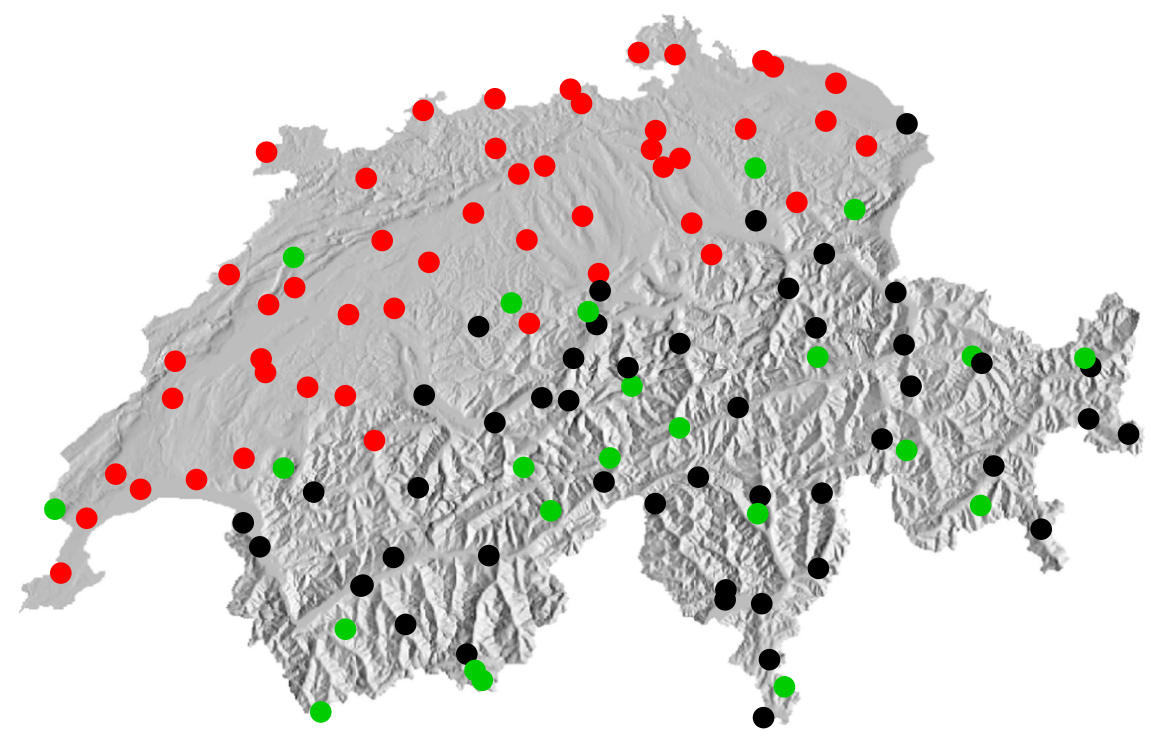

FIG. 5. Communities detected in the network constructed before the STL decomposition. 


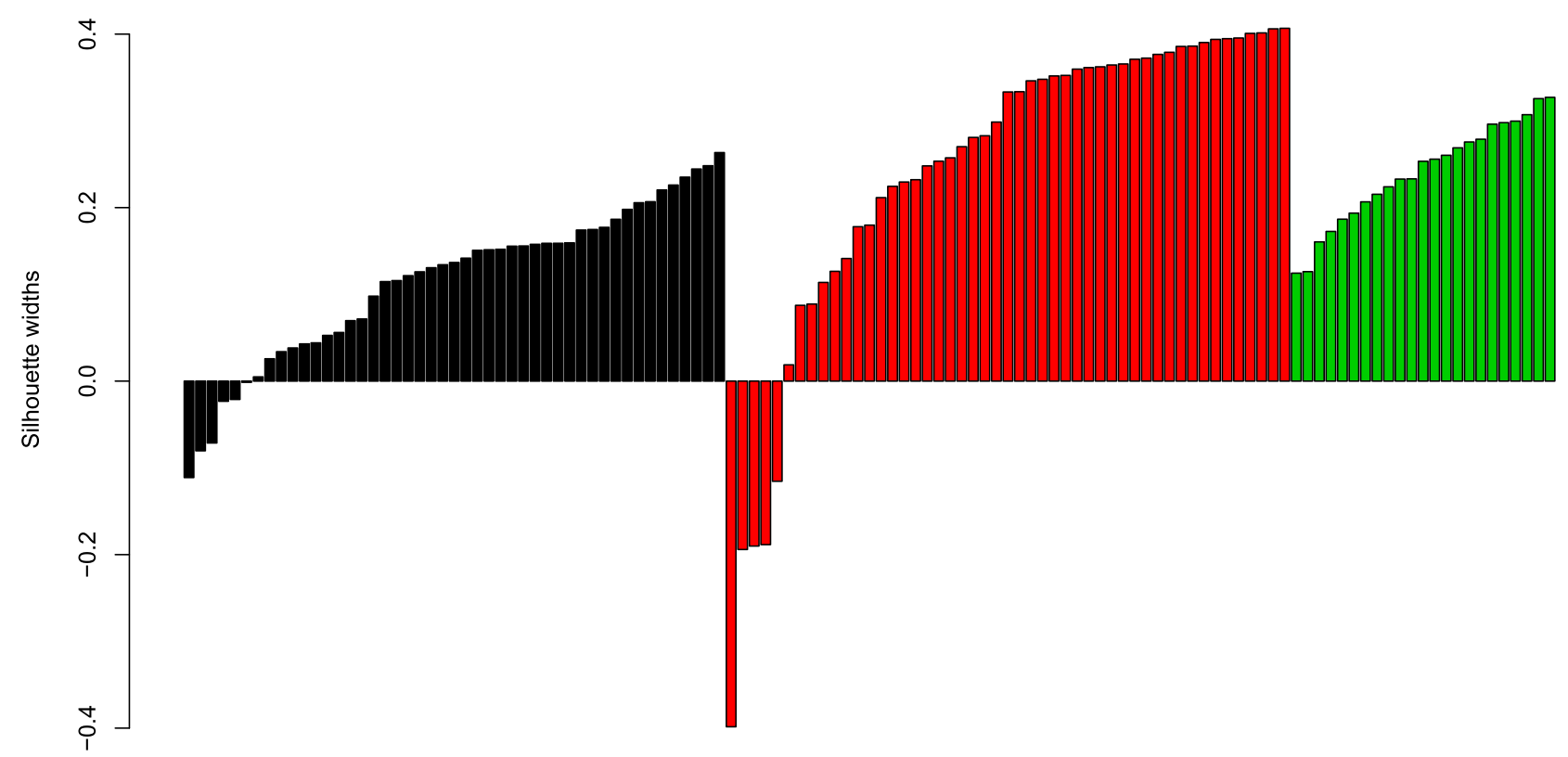

FIG. 6. Silhouette width of each node of each community obtained, on the Mutual information matrix, before applying the STL decomposition. The average values of the silhouette widths are 0.12 for the first community (black), 0.24 for the second community (red), and 0.24 for the third community (green). The total average is 0.19 .

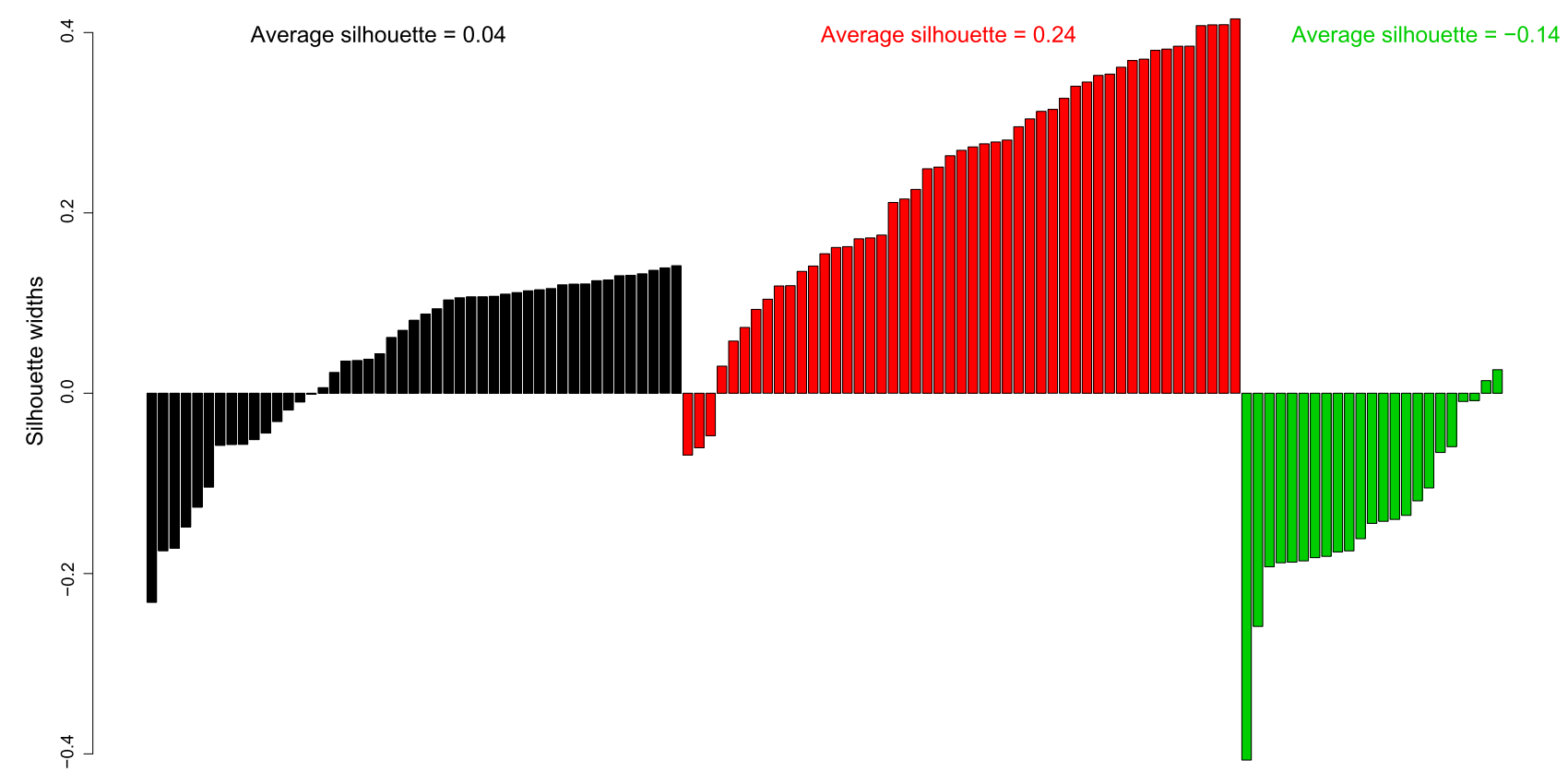

FIG. 7. Silhouette width of each node of each community obtained, on the XY coordinates, before applying the STL decomposition. The average values of the silhouette widths are 0.04 for the first community (black), 0.24 for the second community (red), and -0.14 for the third community (green). The total average is 0.09 . 
WFJ

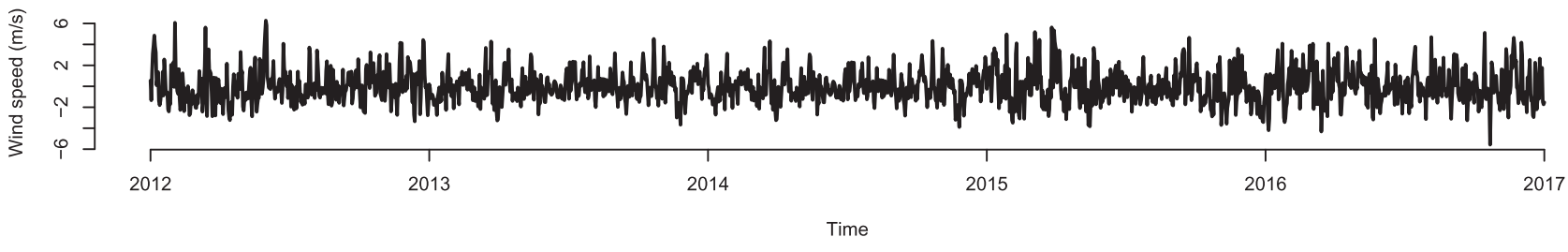

KOP

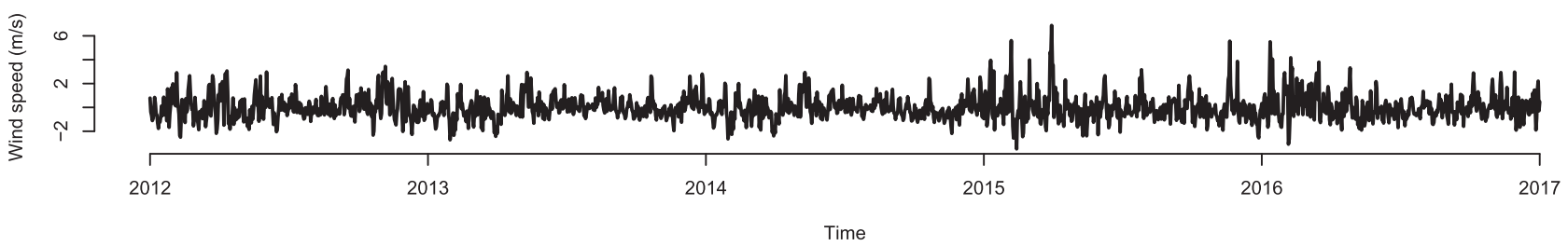

BAS

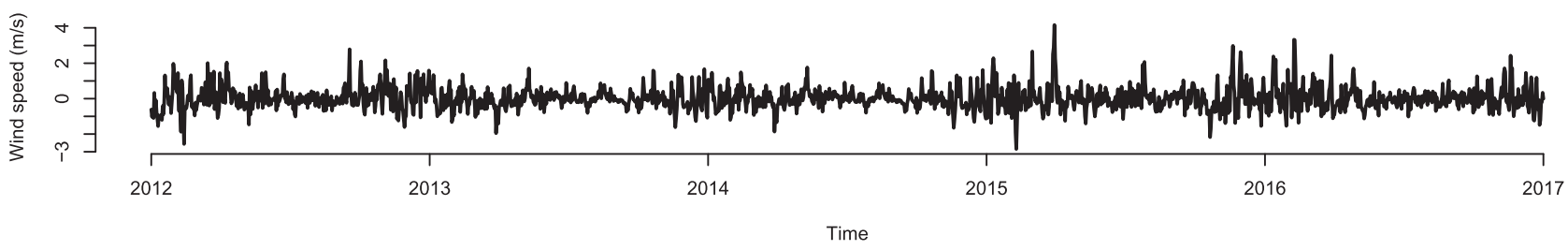

$\mathrm{CHU}$

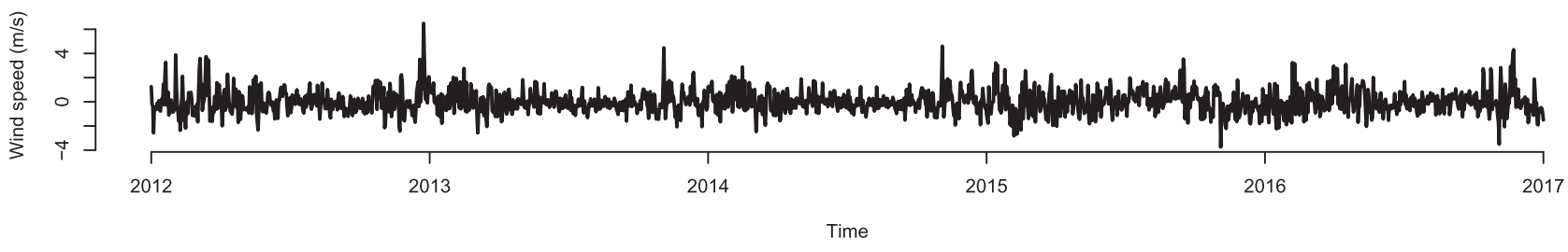

LUG

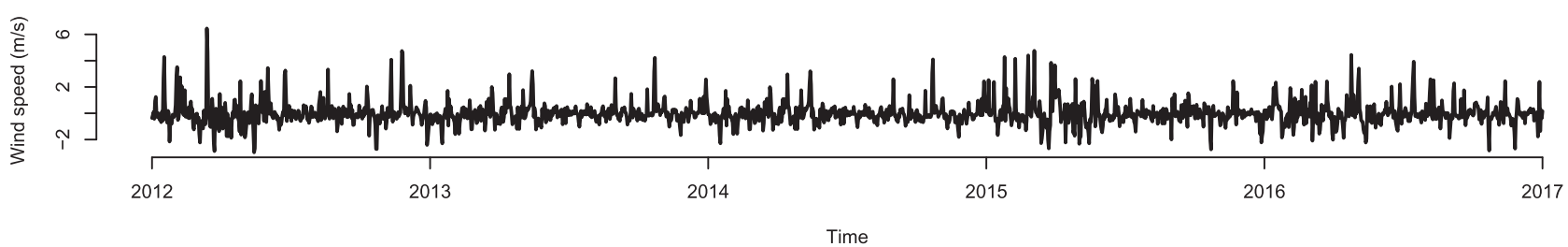

FIG. 8. Residuals of wind series, shown in Fig. 2, obtained by using the STL decomposition.

R library $\left.{ }^{30}\right)$. Then, we applied the community detection MultiLevel method to the residual wind series. Figure 8 shows the residuals of the same time series shown in Fig. 2, and Fig. 9 presents two detected communities.
Mapping the communities on the Swiss territory, the two communities do not show significant spatial mixing (Fig. 10). Furthermore, the silhouette width for each station of each community is shown in Figs. 11 and 12, and the mean silhouette values are 


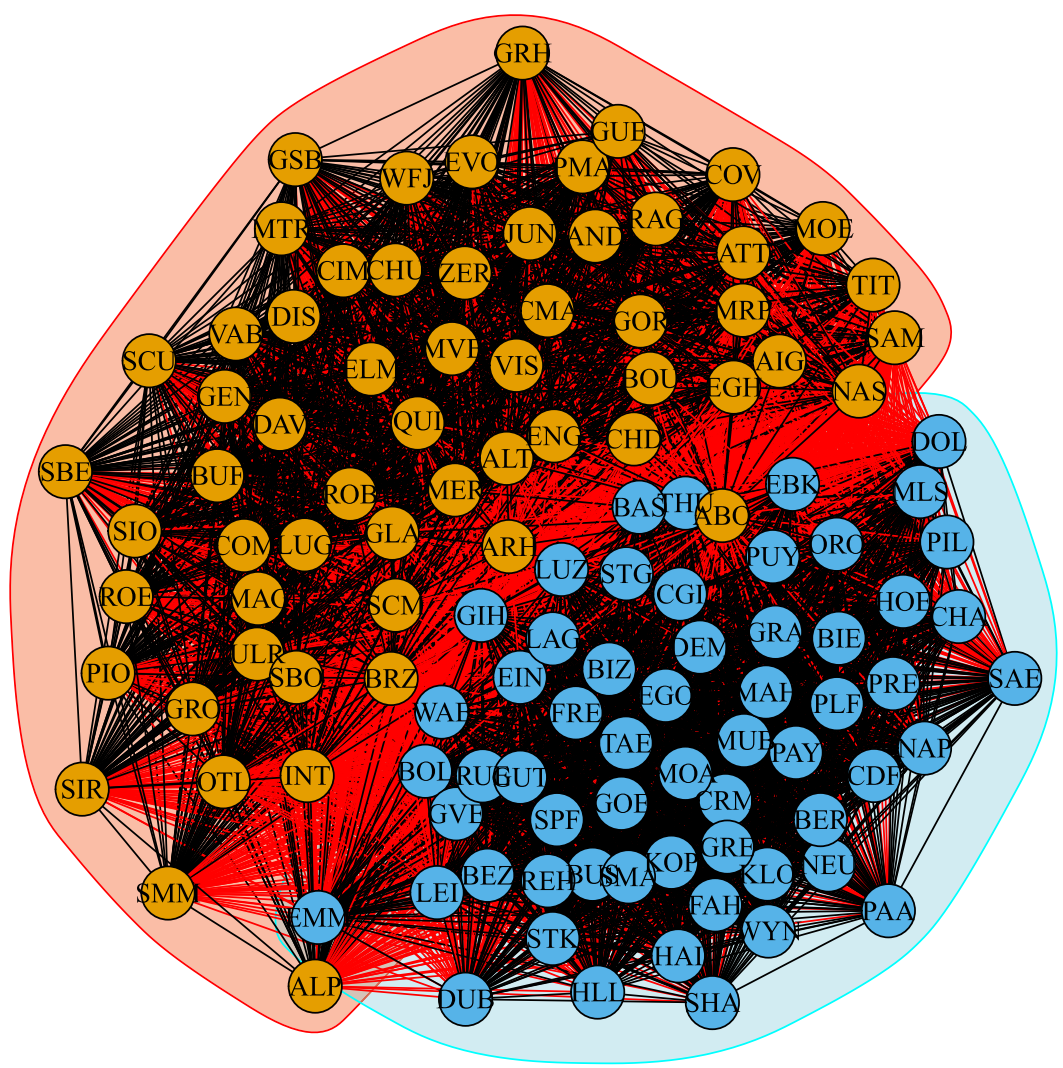

FIG. 9. Network visualisation with the two communities obtained by the ML method after applying the STL.

0.35 (mutual information matrix) and 0.24 (XY coordinates), respectively. These values are better than those obtained on the original data before applying STL. This indicates that there is no significant spatial mixing between the two communities. This result was also found significant comparing it with the silhouette widths calculated for 1000 random spatial distribution of the stations. Figures 13 and 14 show the histogram of the silhouette width for the randomised classes.

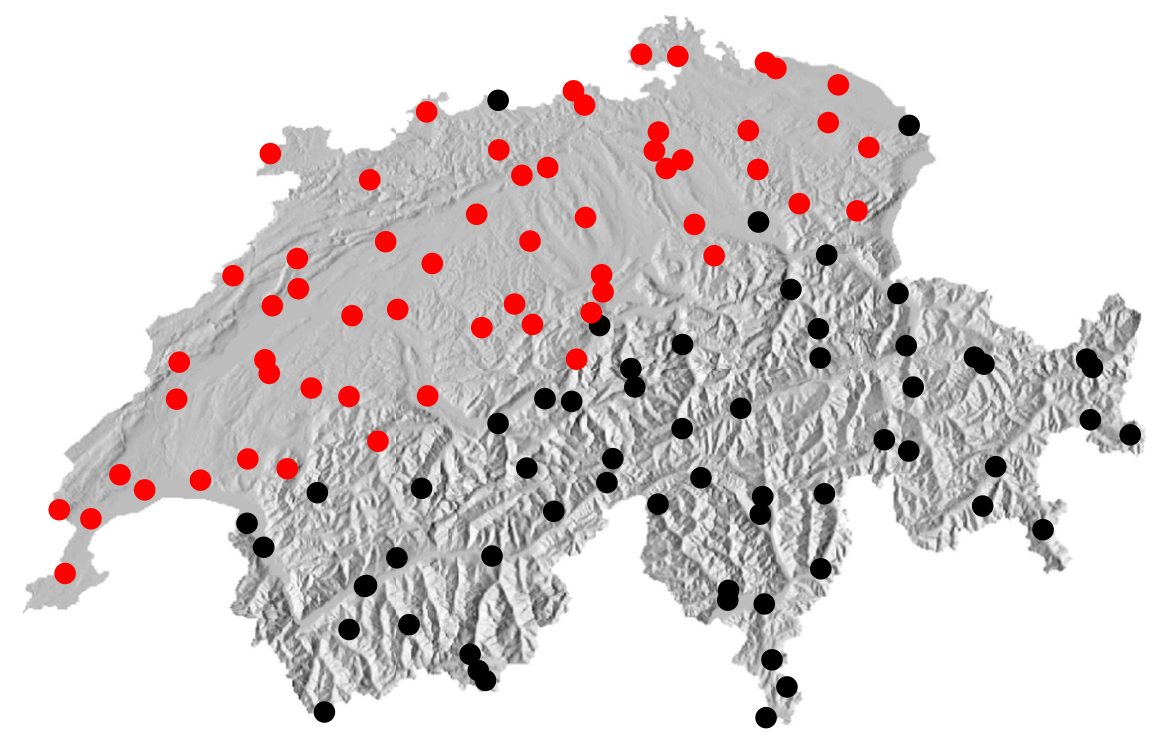

FIG. 10. Communities detected in the network constructed after the STL decomposition. 


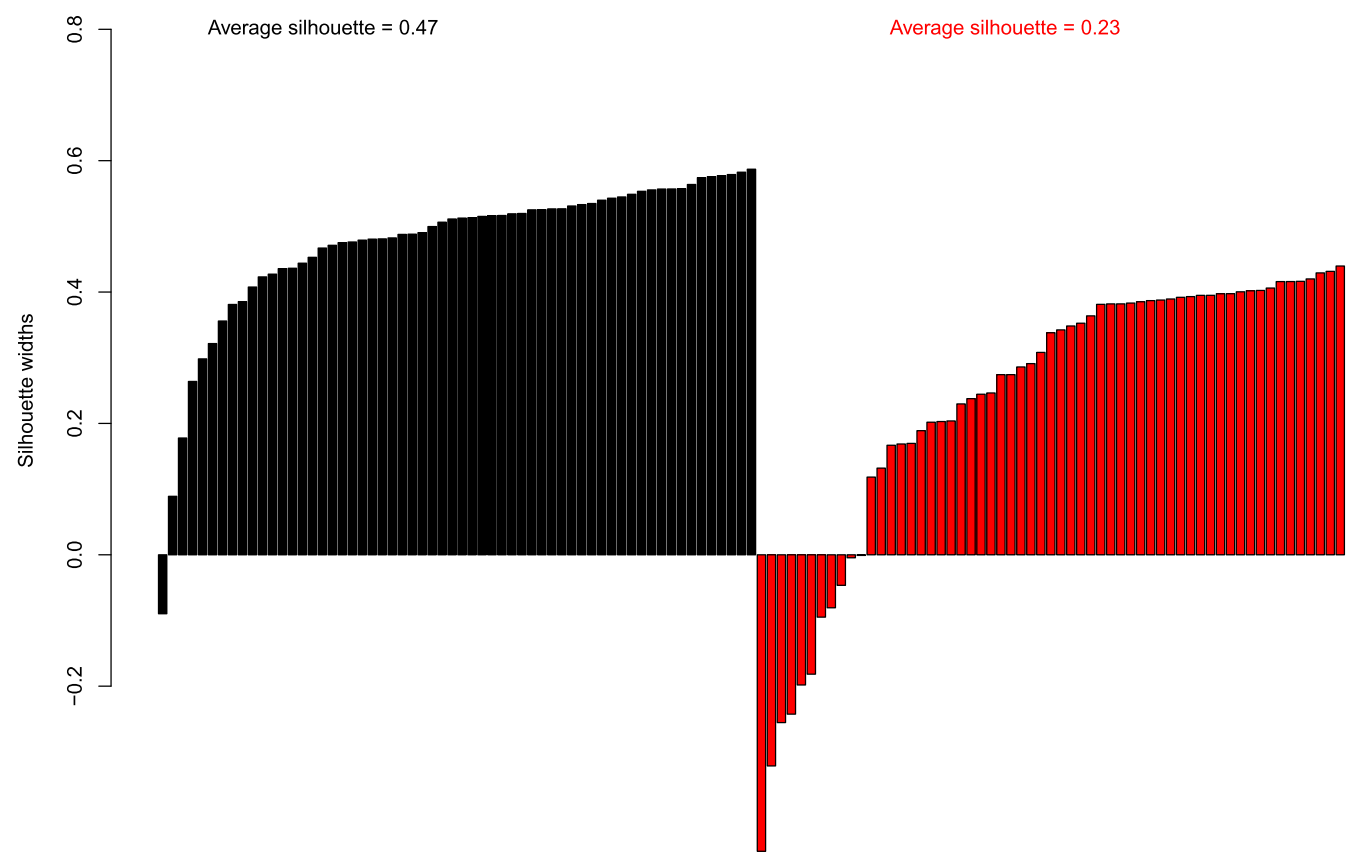

FIG. 11. Silhouette width of each node of each community obtained, on the mutual information matrix, after applying the STL decomposition. The average values of the silhouette widths are 0.47 for the first community (black), 0.23 for the second community (red). The total average is 0.35 .

By using the STL, the global trend and the yearly cycle are removed from each time series. The residual of each wind time series could represent the inner fluctuations of the wind speeds at each location, which could indicate more properly the morphotopographic and climatic characteristics of each measuring site. In addition, wind speeds generally vary depending on the pressure height contours. ${ }^{43}$ Moreover, Weber et al. studied wind climatology in Switzerland and discussed links between founded

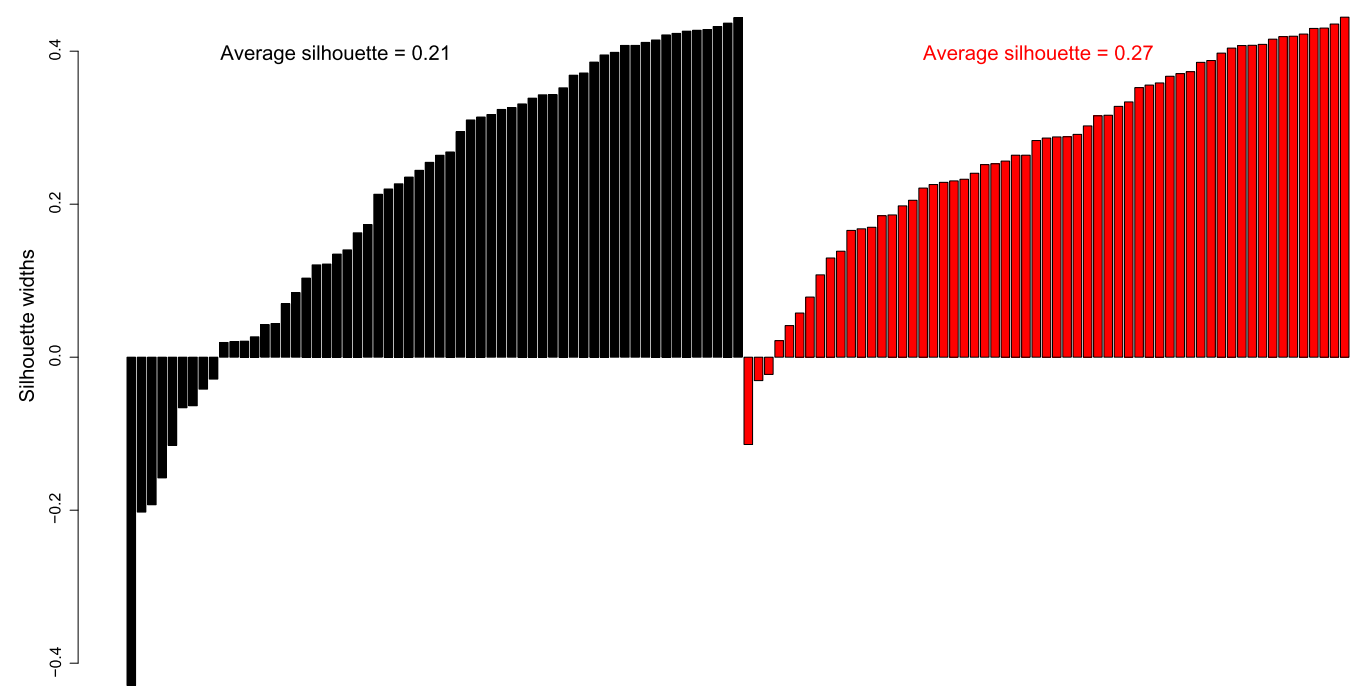

FIG. 12. Silhouette width of each node of each community obtained, on the $X Y$ coordinates, after applying the STL decomposition. The average values of the silhouette widths are 0.21 for the first community (black), 0.27 for the second community (red). The total average is 0.24 . 


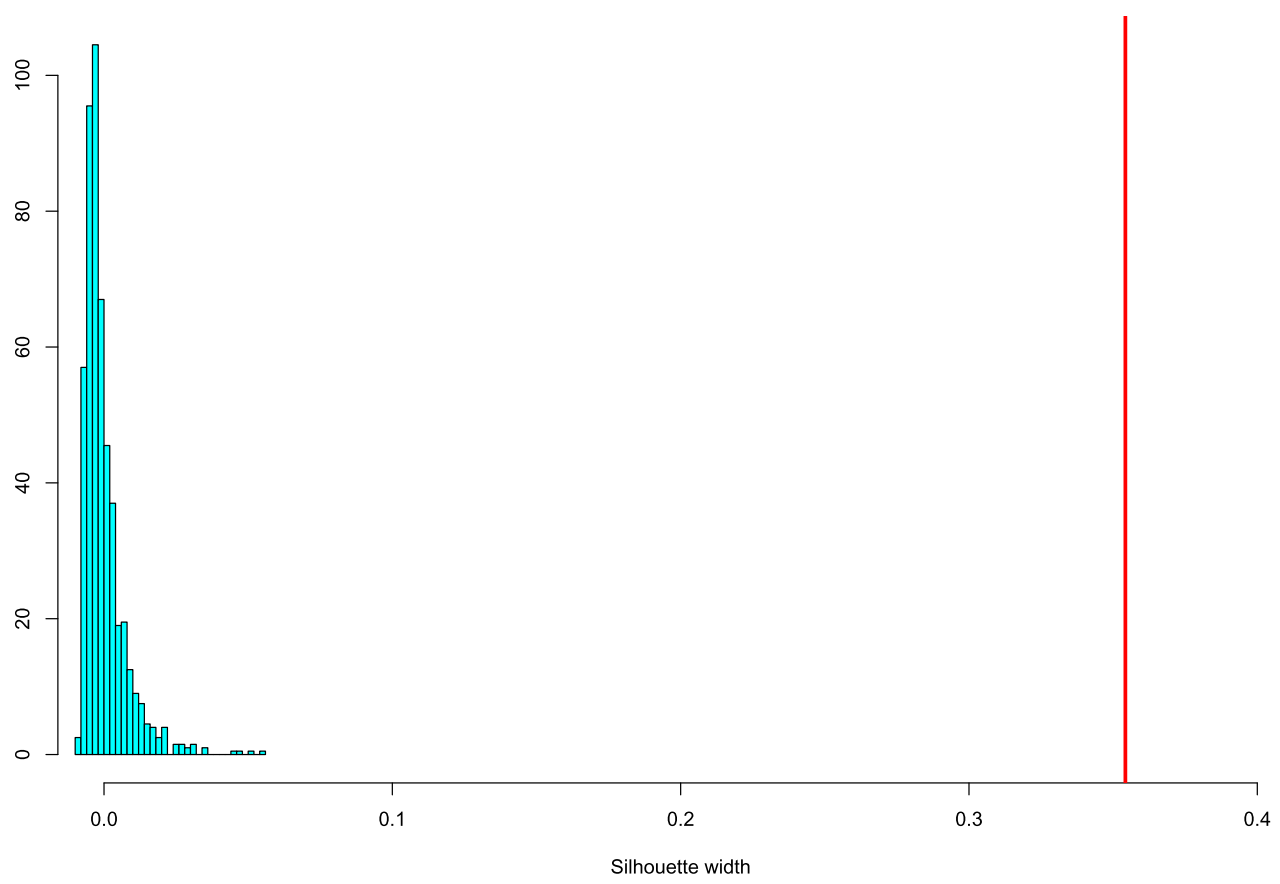

FIG. 13. Comparison between the silhouette width (obtained using the mutual information matrix) histogram of 1000 random classes (blue) and the total average silhouette width for classes obtained after the STL decomposition (red).

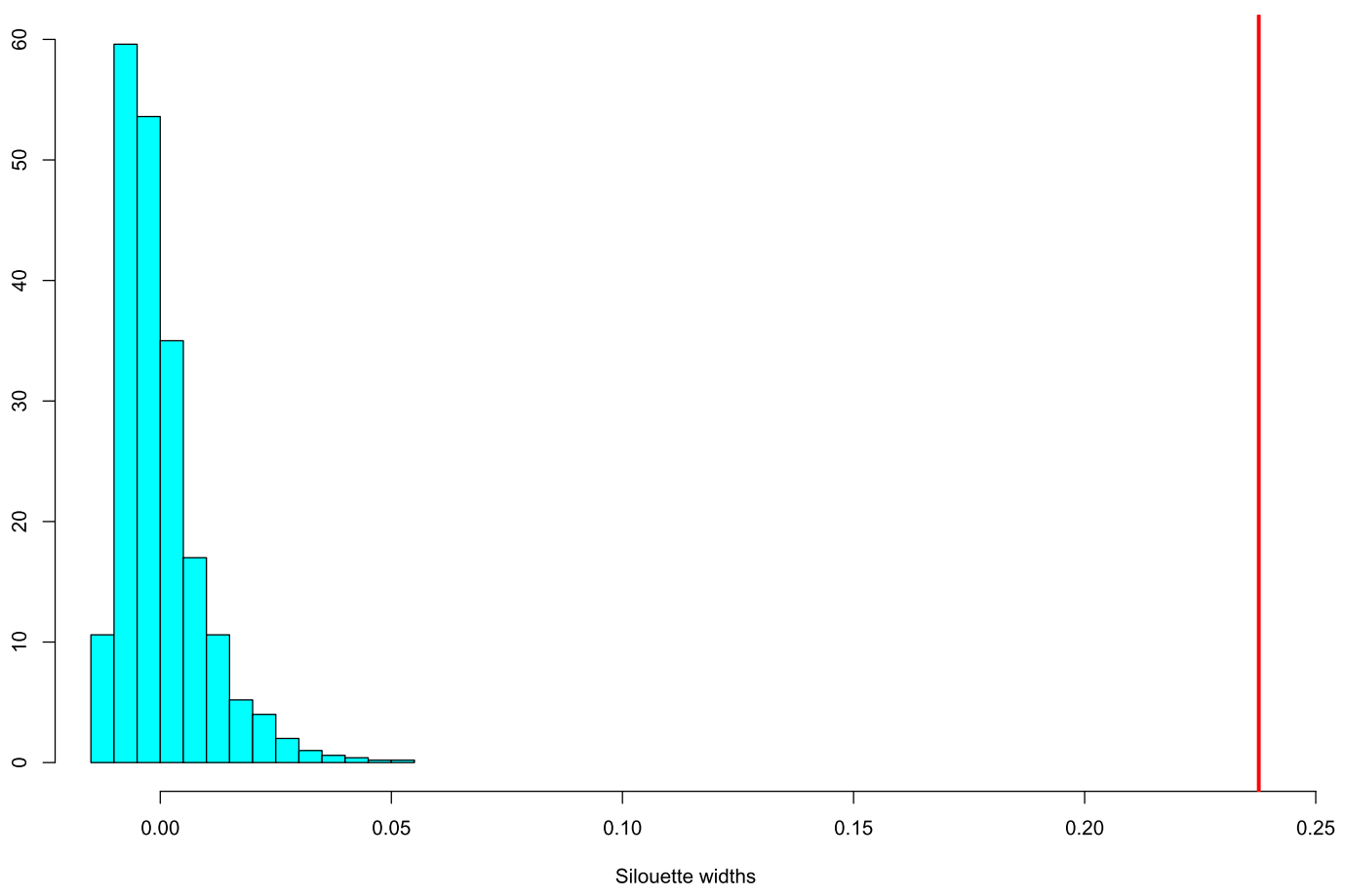

FIG. 14. Comparison between the silhouette width (obtained using the XY coordinates) histogram of 1000 random classes (blue) and the total average silhouette width for classes obtained after the STL decomposition (red). 
classes and pressure. ${ }^{42}$ They also found that the characteristics of these patterns are also provided by a wind rose, which is defined by the atmospheric processes and complex orography in Switzerland. ${ }^{42}$

The presented results are consistent with the previous work, ${ }^{21}$ in which the authors performed the spatial mapping of the multifractal parameters on the wind speed in Switzerland, and they found a canalization effect that separates these regions.

\section{CONCLUSIONS}

1. The wind network, constructed by representing the interactions between the nodes using the mutual information, highlights the (nonlinear) correlations among the wind series.

2. The STL decomposition permits one to extract the residuals of the wind speed not influenced by the trends and annual weather-induced forcing, but only by local meteoclimatic features depending on the geomorphological and topographic properties of each measuring station.

3. The MultiLevel method for the community detection in the mutual information-based network of wind series shows different topological structures of the monitoring system, before and after the removal of the trend and seasonal components. The network constructed on the original data is characterized by three different communities, while that constructed on the residual data (deprived of the trend and seasonal component) is characterized only by two communities.

4. The communities of the network built on the original data are quite spatially mixed. However, the communities of the network built on the residual data are, instead, spatially well separated, with no significantly apparent mixing between the stations belonging to the two communities.

5. The silhouette width, used to quantify the spatial mixing between the found communities, shows an average value for the communities detected in the network based on the original data much lower than that found for the communities detected in the network based on the residuals. Furthermore, the last is significant against the silhouette widths calculated after shuffling the stations of the two communities.

6. The two communities detected after removing the trend and seasonal components match very well with climatic zones of Switzerland, the Alps, and the Jura-Plateau. This suggests the potential of the complex network method in disclosing the inner interactions among wind speed series measured in different climatic regions mainly due to the local topographic factors.

\section{ACKNOWLEDGMENTS}

F. Guignard and M. Kanevski thank the support of the National Research Programme 75 "Big Data" (PNR75) of the Swiss National Science Foundation (SNSF). L. Telesca thanks the support of the "Scientific Exchanges" Project No. 180296 funded by the SNSF. M. Laib thanks the support of "Société Académique Vaudoise" (SAV) and the Swiss Government Excellence Scholarships. The authors thank MeteoSwiss for providing the data. They are also grateful to the anonymous reviewers for their constructive comments that contributed to improving the paper.

\section{REFERENCES}

${ }^{1}$ A. Arenas, J. Duch, A. Fernandez, and S. Gomez, "Size reduction of complex networks preserving modularity," New J. Phys. 9, 176 (2007).

${ }^{2}$ V. D. Blondel, J.-L. Guillaume, R. Lambiotte, and E. Lefebvre, "Fast unfolding of communities in large networks," J. Stat. Mech. Theory Exp. 10, P10008 (2008).

${ }^{3}$ S. Boccaletti, V. Latora, Y. Moreno, M. Chavez, and D.-U. Hwang, "Complex networks: Structure and dynamics," Phys. Rep. 424, 175-308 (2006).

${ }^{4} \mathrm{G}$. Cao, Q. Zhang, and Q. Li, "Causal relationship between the global foreign exchange market based on complex networks and entropy theory," Chaos Solitons Fractals 99, 36-44 (2017).

${ }^{5}$ A. Clauset, M. E. J. Newman, and C. Moore, "Finding community structure in very large networks," Phys. Rev. E 70, 066111 (2004).

${ }^{6}$ R. B. Cleveland, W. S. Cleveland, J. E. McRae, and I. Terpenning, "STL: A seasonaltrend decomposition procedure based on Loess," J. Off. Stat. 6, 3-73 (1990).

${ }^{7}$ T. M. Cover and J. A. Thomas, Elements of Information Theory, 2nd ed. (WileyInterscience, 2006), p. 774.

${ }^{8}$ G. D'Amico, F. Petroni, and F. Prattico, "Wind speed prediction for wind farm applications by extreme value theory and copulas," Wind Eng. Ind. Aerodyn. J. 145, 229-236 (2015).

${ }^{9}$ J. F. Donges, Y. Zou, N. Marwan, and J. Kurths, "The backbone of the climate network," Europhys. Lett. 87, 48007 (2009).

${ }^{10}$ J. F. Donges, Y. Zou, N. Marwan, and J. Kurths, "Complex networks in climate dynamics,” Eur. Phys. J. Spl. Top. 174, 157-179 (2009).

${ }^{11}$ R. V. Donner, M. Lindner, L. Tupikina, and N. Molkenthin, "Characterizing flows by complex network methods," in A Mathematical Modeling Approach from Nonlinear Dynamics to Complex Systems, edited by E. E. N. Macau (Springer International Publishing, Cham, 2019), pp. 197-226.

${ }^{12}$ R. V. Donner, M. Wiedermann, and J. F. Donges, "Complex network techniques for climatological data analysis," in Nonlinear and Stochastic Climate Dynamics, edited by C. L. E. Franzke and T. J. O'Kane (Cambridge University Press, 2017), pp. 159-183.

${ }^{13}$ L. Fortuna, S. Nunnari, and G. Guariso, "Fractal order evidences in wind speed time series," in ICFDA'14 International Conference on Fractional Differentiation and Its Applications 2014 (Catania, 2014), pp. 1-6.

${ }^{14} \mathrm{~S}$. Fortunato, "Community detection in graphs," Phys. Rep. 486, 75-174 (2010).

${ }^{15}$ A. P. Garcia-Marin, F. J. Jiménez-Hornero, and J. L. Ayuso-Munoz, "Multifractal analysis of validated wind speed time series," Chaos 23, 013133 (2013).

${ }^{16}$ M. Girvan and M. E. J. Newman, "Community structure in social and biological networks,” Proc. Natl. Acad. Sci. U.S.A. 99, 7821-7826 (2002).

${ }^{17}$ A. Gozolchiani, K. Yamasaki, O. Gazit, and S. Havlin, "Pattern of climate network blinking links follows el Niño events," Europhys. Lett. 83, 28005 (2008).

${ }^{18} \mathrm{~K}$. Holger and N. K. Vitanov, "Markov chain model for turbulent wind speed data," Physica A 342, 315-321 (2004), Proceedings of the VIII Latin American Workshop on Nonlinear Phenomena.

${ }^{19}$ A. Jiménez, A complex network model for seismicity based on mutual information," Physica A 392, 2498-2506 (2013).

${ }^{20}$ M. Kim and H. Sayama, "Predicting stock market movements using network science: An information theoretic approach,” Appl. Netw. Sci. 2, 35 (2017).

${ }^{21}$ M. Laib, J. Golay, L. Telesca, and M. Kanevski, "Multifractal analysis of the time series of daily means of wind speed in complex regions," Chaos Solitons Fractals 109, 118-127 (2018).

${ }^{22}$ M. Laib, L. Telesca, and M. Kanevski, "Long-range fluctuations and multifractality in connectivity density time series of a wind speed monitoring network," Chaos 28, 033108 (2018).

${ }^{23}$ M. Laib, L. Telesca, and M. Kanevski, "Periodic fluctuations in correlation-based connectivity density time series: Application to wind speed-monitoring network in Switzerland," Physica A 492, 1555-1569 (2018).

${ }^{24}$ A. Lancichinetti, S. Fortunato, and F. Radicchi, "Benchmark graphs for testing community detection algorithms," Phys. Rev. E 78, 046110 (2008).

${ }^{25} \mathrm{M}$. Newman, "The structure and function of complex networks," SIAM Rev. 45, 167-256 (2003).

${ }^{26}$ M. E. J. Newman, “Analysis of weighted networks," Phys. Rev. E 70, 056131 (2004). 
${ }^{27}$ M. de Oliveira Santos, T. Stosic, and B. D. Stosic, "Long-term correlations in hourly wind speed records in Pernambuco, Brazil," Physica A 391, 1546-1552 (2012).

${ }^{28}$ J. O. Pierini, M. Lovallo, and L. Telesca, "Visibility graph analysis of wind speed records measured in central Argentina," Physica A 391, 5041-5048 (2012).

${ }^{29} \mathrm{P}$. Pons and M. Latapy, "Computing communities in large networks using random walks," in Computer and Information Sciences-ISCIS 2005, edited by P. Yolum, T. Güngör, F. Gürgen, and C. Özturan (Springer, Berlin, Heidelberg, 2005), pp. 284-293.

${ }^{30} \mathrm{R}$ Core Team, R: A Language and Environment for Statistical Computing, R Foundation for Statistical Computing, Vienna, Austria (2018).

${ }^{31}$ J. Reichardt and S. Bornholdt, "Statistical mechanics of community detection," Phys. Rev. E 74, 016110 (2006).

${ }^{32} \mathrm{M}$. Rosvall and C. T. Bergstrom, "An information-theoretic framework for resolving community structure in complex networks," Proc. Natl. Acad. Sci. U.S.A. 104, 7327-7331 (2007).

${ }^{33}$ P. J. Rousseeuw, "Silhouettes: A graphical aid to the interpretation and validation of cluster analysis," J. Comput. Appl. Math. 20, 53-65 (1987).

${ }^{34} \mathrm{~K}$. Steinhaeuser, N. V. Chawla, and A. R. Ganguly, "An exploration of climate data using complex networks," in Proceedings of the Third International Workshop on Knowledge Discovery from Sensor Data (ACM, 2009), pp. 23-31.
${ }^{35} \mathrm{~L}$. Telesca, M. Lovallo, and M. Kanevski, "Power spectrum and multifractal detrended fluctuation analysis of high-frequency wind measurements in mountainous regions," Appl. Energy 162, 1052-1061 (2016).

${ }^{36}$ N. A. Treiber, J. Heinermann, and O. Kramer, "Wind power prediction with machine learning," in Computational Sustainability (Springer International Publishing, 2016), pp. 13-29.

${ }^{37} \mathrm{~A}$. Tsonis and P. Roebber, "The architecture of the climate network," Physica A 333, 497-504 (2004)

${ }^{38}$ A. A. Tsonis and K. L. Swanson, "Topology and predictability of el Niño and la Niña networks," Phys. Rev. Lett. 100, 228-502 (2008).

${ }^{39}$ A. A. Tsonis, K. L. Swanson, and P. J. Roebber, "What do networks have to do with climate?,” Bull. Am. Meteorol. Soc. 87, 585-595 (2006).

${ }^{40} \mathrm{M}$. P. Wand, "Fast computation of multivariate kernel estimators," J. Comput. Graph. Stat. 3, 433-445 (1994)

${ }^{41}$ M. P. Wand and M. C. Jones, Kernel Smoothing (Chapman \& Hall, 1995), p. 212.

${ }^{42} \mathrm{O}$. R. Weber and M. Furger, "Climatology of near-surface wind patterns over Switzerland," Int. J. Climatol. 21, 809-827 (2001).

${ }^{43} \mathrm{C}$. D. Whiteman, Mountain Meteorology: Fundamentals and Applications (Oxford University Press, 2000), p. 372.

${ }^{44} \mathrm{~K}$. Yamasaki, A. Gozolchiani, and S. Havlin, "Climate networks around the globe are significantly affected by el Niño," Phys. Rev. Lett. 100, 228501 (2008).

${ }^{45} \mathrm{Z}$. Yang, R. Algesheimer, and C. J. Tessone, "A comparative analysis of community detection algorithms on artificial networks," Sci. Rep. 6, 30750 (2016). 